\title{
Corrigendum to:
}

\section{"Aspherical four-manifolds and the centres of two-knot groups"}

\author{
Jonathan A. Hillman
}

M. N. Dyer has pointed out that the proof of the key Lemma on "hopfian" rings in $\$ 2$ of [1] is incorrect. As I have been unable to find a correct argument, the results on pages $465-469$ are moot. (Corollaries 2 and 3 on page 470 are true as it is easy to see that the lemma holds for any commutative ring, while the results in $\$ 5$ use only Kaplansky's original theorem, and not the lemma.) I hope that some ring-theorist may be able to prove the lemma.

In the first line of page 469 , the map from $H^{2}\left(C^{*}\right)$ to $\operatorname{Hom}_{\Gamma}\left(H_{2}\left(C_{*}\right), \Gamma\right)$ given by the universal coefficient spectral sequence is, a priori, only a monomorphism. However the theorem is still true (without any essential change in the argument), modulo the lemma.

A. Suciu has pointed out that the map $\Phi$ in line 1 of page 471 should be replaced by its square $\Phi^{2}$, to ensure that the mapping torus $M$ be orientable.

I am grateful to Dyer and Suciu for their observations.

\section{REFERENCE}

[1] Hillman, J. A., Aspherical four-manifolds and the centres of two-knot groups, Commentarii Math. Helveticii 56 (1981), 465-473.

Department of Mathematics

The University of Texas

Austin, Texas 78712, USA

Received October 20, 1982 